\title{
TED: A Pretrained Unsupervised Summarization Model with Theme Modeling and Denoising
}

\author{
Ziyi Yang ${ }^{1 *}$, Chenguang Zhu ${ }^{2 *}$, Robert Gmyr ${ }^{2}$, Michael Zeng ${ }^{2}$, Xuedong Huang ${ }^{2}$, Eric Darve ${ }^{1}$ \\ Stanford University ${ }^{1}$ \\ \{zy99, darve\}estanford.edu \\ Microsoft Cognitive Services Research Group ${ }^{2}$ \\ $\{$ chezhu, rogmyr, nzeng, $x d h\} @ m i c r o s o f t . c o m$
}

\begin{abstract}
Text summarization aims to extract essential information from a piece of text and transform the text into a concise version. Existing unsupervised abstractive summarization models leverage recurrent neural networks framework while the recently proposed transformer exhibits much more capability. Moreover, most of previous summarization models ignore abundant unlabeled corpora resources available for pretraining. In order to address these issues, we propose TED, a transformerbased unsupervised abstractive summarization system with pretraining on large-scale data. We first leverage the lead bias in news articles to pretrain the model on millions of unlabeled corpora. Next, we finetune TED on target domains through theme modeling and a denoising autoencoder to enhance the quality of generated summaries. Notably, TED outperforms all unsupervised abstractive baselines on NYT, CNN/DM and English Gigaword datasets with various document styles. Further analysis shows that the summaries generated by TED are highly abstractive, and each component in the objective function of TED is highly effective.
\end{abstract}

\section{Introduction}

Summarization refers to the task of condensing a document into a shorter version without losing the key information. Summarization models can be categorized into two types: abstractive and extractive. Extractive models select sentences from the input article as the summary. Such process ensures a basic level of grammaticality and accuracy, but also limits the model ability to copying. In contrast, abstractive models summarize a document using newly generated tokens and phrases that may not be found in the original article, which involves

* Equal contribution. Work was done during first author's internship at Microsoft. a process requiring an advanced ability to refine, paraphrase and re-organize language information (See et al., 2017; Narayan et al., 2018; Gunel et al., 2020).

Like most machine learning algorithms, summarization models can also be divided into supervised and unsupervised categories. Supervised approaches require in-domain parallel data, i.e. both input articles and corresponding reference summaries must be present for the teacher-forcing training (Hermann et al., 2015; Liu and Lapata, 2019). Unfortunately, high-quality paired data are not always available across different text domains and styles. Moreover, considering the fact that summarization is not an easy task even for people, reliable human-labeled data are also difficult to obtain. Therefore, several unsupervised summarization approaches have been proposed, which do not require reference summaries for the target domain. We introduce these methods as follows.

Unsupervised extractive models. TextRank (Mihalcea and Tarau, 2004) encodes sentences in the article as nodes in an undirected graph. The weights of edges are measured by sentences similarity. The centrality of a node (sentence) is computed by PageRank (Brin and Page, 1998) to decide whether a sentence should be included in the final summary. Zheng and Lapata (2019) advances upon TextRank by encoding sentences with BERT representation (Devlin et al., 2018) to compute pairs similarity and build graphs with directed edges decided by the relative positions of sentences.

Unsupervised abstractive models. Baziotis et al. (2019) leverages differentiable sampling and optimizes by re-constructing the input article from the generated summary. Chu and Liu (2018) proposes a similar idea in the multi-document summarization setting. Wang and Lee (2018) uses adversarial training and reinforcement learning to make the summary human-readable. Févry and 
Phang (2018) adopts denoising autoencoders originally used in sentence compression. However, most of these models are only tested on datasets with considerably small article/summary length. Also, previous models usually utilize the recurrent neural networks (RNNs). However, transformers (Vaswani et al., 2017; Devlin et al., 2018) have shown superior performances over RNNs on various NLP tasks, including machine translation, reading comprehension, sentiment analysis, etc. Few Efforts have been made to leverage transformers in unsupervised abstractive summarizations.

Pretraining Language Model. In recent years, pretraining language models have proved to be quite powerful in solving numerous NLP tasks. The state-of-the-art pretrained models include $\mathrm{CoVe}$ (McCann et al., 2017), ELMo (Peters et al., 2018), GPT (Radford et al., 2018), BERT (Devlin et al., 2018) and UniLM (Dong et al., 2019). Taking advantage of corpora with billions of tokens, the pretrained language models learn universal and robust representations for various semantic structures and linguistic relationships. As a result, pretrained models have been widely used with considerable success in applications such as question answering (Zhu et al., 2018), sentiment analysis (Peters et al., 2018) and passage reranking (Nogueira and Cho, 2019). Furthermore, UniLM (Dong et al., 2019) leverages its sequence-to-sequence capability for abstractive summarization; the BERT model has been employed as an encoder in BERTSUM (Liu and Lapata, 2019) for supervised extractive and abstractive summarization.

In this paper, we present TED, a pretrained unsupervised abstractive summarization model which is finetuned with theme modeling and denoising on in-domain data. TED utilizes a transformer-based encoder-decoder structure and the pretraining leverages large-scale corpora containing millions of unlabeled articles. Our primary contributions are twofold as follows.

First, we leverage the lead bias in news articles to pretrain TED. The lead bias is introduced by the journalistic convention of writing using an inverted pyramid structure, placing the most important information in the beginning of an article. We propose to use the leading sentences as the target summary and train the model to predict it during pretraining. In this way, we pretrain a summarization model on a large-scale corpus with $21.4 \mathrm{M}$ news articles. The model yields better performance than most existing unsupervised methods.

Second, to finetune on specific datasets, TED is further trained with a theme modeling loss and a denoising autoencoder. The role of the theme modeling module is to make the generated summary semantically close to the article. The module uses a semantic classifier trained using a discriminative objective function. Furthermore, to optimize on the generated summary tokens, we adopt the Gumbel-Softmax (Jang et al., 2016) estimator to replace the non-differentiable arg max. The denoising autoencoder has been previously used in unsupervised machine translation (Lample et al., 2017) and sentence compression (Févry and Phang, 2018), and we employ it to help the model extract salient information from corrupted text.

Instead of classical word tokenization, we adopt the SentencePiece tokenization (Kudo and Richardson, 2018) to alleviates the long-standing out-ofvocabulary $(\mathrm{OOV})$ problem in language generation tasks (Luong et al., 2014; Sennrich et al., 2015). We test TED on several benchmark datasets. The experimental results show that TED outperforms all unsupervised abstractive baselines on all datasets. For example, on the CNN/DM dataset, it outperforms the state-of-the-art unsupervised abstractive model by more than 9 ROUGE-1 points and compares favorably with most unsupervised extractive models. We further show that TED is capable of generating novel words and phrases in summaries and is a highly abstractive system even compared with supervised systems.

\section{Methodology}

In this section, we will go through the model structure of TED, i.e. the transformer encoder and decoder. Then we introduce the pretraining method and two in-domain finetuning objectives: theme modelling and the denoising autoencoder. The overall architecture of TED is illustrated in Fig. 1.

\subsection{Transformer Encoder and Decoder}

Previous unsupervised summarization methods are based on the sequence to sequence (seq2seq) model (Sutskever et al., 2014) that primarily uses the RNN model. As the transformer structure (Vaswani et al., 2017) has been successfully applied in a large number of NLP tasks, TED employs the multi-layer transformer encoder-decoder architecture. We follow the standard transformer design in TED networks and refer readers to Vaswani et al. (2017) 


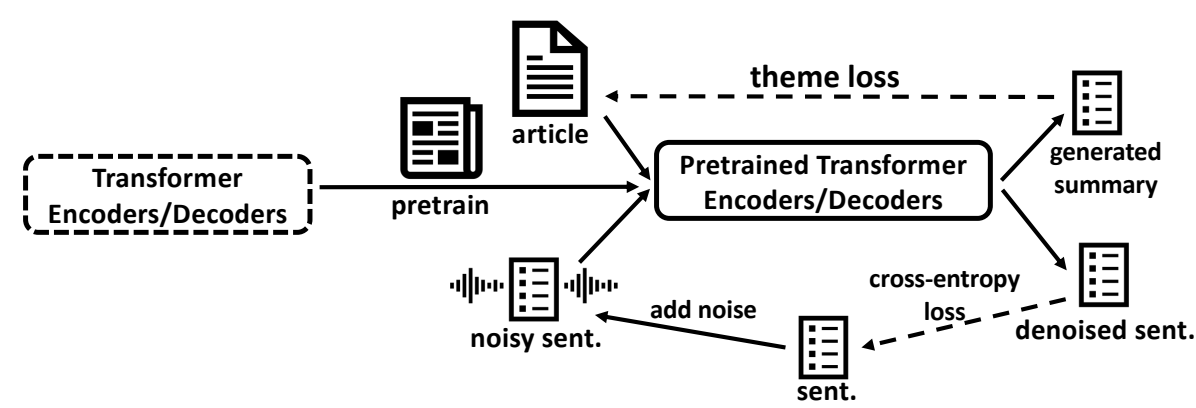

Figure 1: Overall structure of our model. TED first pretrains on news articles and then finetunes with theme modeling and denoising. (from left to right).

for more technical details on transformers. Denote the number of layers (i.e., Transformer blocks) as $L$, the number of self-attention heads as $H$ and the hidden size as $N$. We explore two different configurations in experiments, 4 layers 4 heads $(4 \mathrm{~L} 4 \mathrm{H})$ with $N=512$ and 10 layers 8 heads (10L8H) with $N=720$.

Denote the input article tokens sequence as $X=\left\{x_{1}, x_{2}, \ldots, x_{n}\right\}$, and each token is first transferred to a vector by a trainable embeddings matrix $\boldsymbol{V}$. The output from transformer encoder $E$ is a sequence of encoded vectors $E(X)=$ $\left\{\boldsymbol{u}_{1}^{E}, \boldsymbol{u}_{2}^{E}, \ldots, \boldsymbol{u}_{n}^{E}\right\}$. The decoder can be viewed as a conditional language model to generate the summary depending on the generator outputs. Given $k$ input summary tokens $W=\left\{w_{1}, w_{2}, \ldots, w_{k}\right\}$, the cross attention layer in the decoder $D$ attends with encoder outputs $\left\{\boldsymbol{u}_{i}^{E}\right\}_{i=1}^{n}$. The decoder outputs are $D\left(\left\{w_{1}, w_{2}, \ldots, w_{k}\right\}\right)=\left\{\boldsymbol{u}_{1}^{D}, \boldsymbol{u}_{2}^{D}, \ldots, \boldsymbol{u}_{k}^{D}\right\}$. The probability distribution over the vocabulary for $w_{k+1}$ is given by:

$$
P\left(w_{k+1} \mid w_{1: k}, x_{1: n}\right)=\operatorname{softmax}\left(\boldsymbol{V} \boldsymbol{u}_{k}^{D}\right)
$$

In traditional tokenization algorithms, efforts have been made to address the out-of-vocabulary (OOV) issue (Yang et al., 2019) at the cost of losing semantic information, such as mapping OOV words to a special "UNK" token. To mitigate the open vocabulary problem, we adopt SentencePiece (Kudo and Richardson, 2018), a data-driven method that trains tokenization models from sentences in large-scale corpora. The advantage of the SentencePiece model is that its subwords can cover all possible word forms and the subword vocabulary size is controllable. In the evaluation experiments, we train a SentencePiece subword vocabulary of size 32,000 .

Note for supervised summarization models, during training, the inputs to the decoder are the groundtruths/reference summary tokens; for unsupervised learning, input tokens are generated in the previous pass, i.e. one new token is generated in one pass. More details are available in section 2.3.1.

\subsection{Pretraining with Unlabeled Corpora}

Leveraging large scale unlabeled text corpora to pretrain models has been proven as an effective method in multiple NLP tasks (Devlin et al., 2018). However, such approach has not yet been utilized in text summarization.

News articles follow an inverted pyramid structure, i.e. front loading the most salient information. This so-called "lead bias" for news summarization is so strong that See et al. (2017) have shown that using the first 3 sentences in a news article as a summary can score higher than many sophisticated deep learning models. Although this poses a great challenge to previous research, we take advantage of this property in our favor in the pretraining phase of TED.

For a news article, we set the target summary to be the first three sentences. This allows the model to exploit the structural bias of the news domain and infer the most important information using the background materials in the remainder of the article. To collect data for pretraining, we obtain three years of online news articles from 2016 to 2019 via an industrial search engine. The search engine indexes major online news domain, for instance, New York Times and Bloomberg. Then we collect the parsed articles within the 2016-2019 time range as the raw data. Note that this time span does not overlap any of three test datasets we use in this paper, therefore the pretraining should not lead to data leakage in test. It is also worth noting that this idea of utilizing structural bias for largescale summarization pretraining is not limited to 


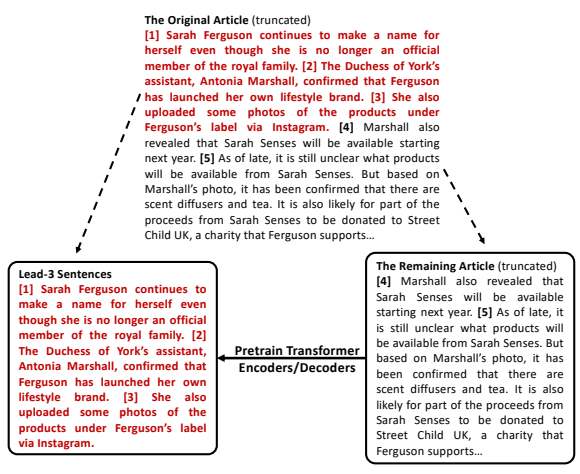

Figure 2: An example of the pretraining task: predict the Lead-3 sentences (as the target summary) using the rest of the article.

specific types of models, and it can be applied to other types of text as well: academic papers with abstracts, novels with editor's notes, books with tables of contents.

However, one should carefully examine and clean the source data to take advantage of lead bias, as the top three sentences may not always form a good summary. Therefore, we conduct strict data cleaning to remove irrelevant distracting content and filter out articles whose top three sentences do not form a good summary:

First, many news articles begin with media names, reporter names, dates or other irrelevant information for summarization, e.g. "New York (CNN) -", "Adam Smith, June 3rd 2018:". We automatically clean these using regular expressions.

Second, we only include articles whose top three sentences contain between 10 and 150 words, and remaining sentences contain between 150 and 1,200 words. The criterion on top three sentences is set to filter out articles with either extremely short leading sentences, e.g. phrases of one or two words, which contain too little information to be reasonable summaries, or exceedingly long leading sentences to reduce the pretraining time. The limit on total number of words in the article is to filter out very long articles to reduce memory consumption. Another purpose is to remove very short articles of which the information is too condensed and not suitable for summarization pretraining.

Third, we also remove articles in which the first three sentences may not contain the major information in the article. We use a simple and easy-tocompute metric: overlapping words. We compute the portion of non-stopping words in the top three sentences that also appear in the rest of an article. A higher ratio indicates that the rest of the article is likely to elaborate on the beginning part. We keep those articles with the ratio of overlapping words higher than 0.65 . We pick this threshold based on observations in the CNN/DM dataset, where the median overlapping ratio of non-stopping words between golden summary and the article is 0.87 , and the median ratio between the top three sentences and the rest of the article is 0.77 . Setting the threshold at 0.65 makes the final training set size fit with the available computation resources and ensures that the leading sentences contain enough information.

Finally, we end up with $21.4 \mathrm{M}$ articles, out of which 12,000 articles are randomly sampled as the validation set. We conduct pretraining for 10 epochs and pick the model with the best ROUGE-L score on the validation set. The pretraining task is to predict to the first three sentences of an article using the rest of the article (so pretraining will not teach the model to simply copy the leading three sentences since they are removed from the input to the transformers). Note that TED does not start off from other pretrained models like Bert.

After pretraining, in order to adapt TED to a specific target dataset (for evaluation), we finetune TED on the target dataset in an unsupervised manner. The finetuning objective functions includes the following: theme modeling and denoising autoencoder.

\subsection{Theme Modeling}

Theme modeling aims to make the generated summary semantically close to the input article. We employ differential sampling to enable optimization on generated summaries and train a classifier to improve the semantic relatedness between the output summary and article.

\subsubsection{Differentiable Sampling}

In order to optimize the transformers using output summaries, we need to make the generation of summary tokens differentiable. Recall the conditional probability distribution of token $w_{k+1}$ is $P\left(w_{k+1} \mid w_{1: k}, x_{1: n}\right)=\operatorname{softmax}\left(\boldsymbol{V} \boldsymbol{u}_{k}^{D}\right)$. Let $\boldsymbol{\pi}$ denote $P\left(w_{k+1} \mid w_{1: k}, x_{1: n}\right)$. One can use arg $\max$ on $\pi$ to obtain the token $w_{k+1}$ in the forward pass, however, it is not differentiable in the gradient back-propagation. Although arg max can be avoided by obtaining the embedding of $w_{k+1}$ as a weighted sum of the vocabulary embeddings $\boldsymbol{V}$, this results in an undesirable gap between the training (weighted sum) and the inference (discrete 
sampling) on the forward pass generation. To solve this issue, we employ the straight-through GumbelSoftmax estimator (Jang et al., 2016) as in Yang et al. (2018); Baziotis et al. (2019). Specifically, the forward pass in training still uses arg max sampling, but for gradient computation, the following Gumbel-Softmax distribution is used as a differentiable approximation for the arg max operation:

$$
\tilde{\boldsymbol{\pi}}_{\boldsymbol{i}}=\frac{\left.\exp \left(\log \left(\boldsymbol{\pi}_{i}\right)+g_{i}\right) / \tau\right)}{\left.\sum_{j=1}^{k} \exp \left(\log \left(\boldsymbol{\pi}_{j}\right)+g_{j}\right) / \tau\right)}
$$

where $g_{1}, \cdots, g_{k}$ are i.i.d samples drawn from the Gumbel distribution $G(0,1)$ and $\tau$ denotes the softmax temperature. As shown in Jang et al (2016), as $\tau \rightarrow 0$, the Gumbel-Softmax distribution converges to the categorical (one-hot) distribution; as $\tau \rightarrow$ inf, the Gumbel-Softmax distribution converges to the uniform distribution. Although this gradient estimator is biased, we find that this method works well in practice. We choose $\tau=0.1$ based on the CNN/DM validation set and use this value in all the experiments. Denote the input article as $\boldsymbol{d}$, the generated summary as $\boldsymbol{s}=\left\{w_{1}, w_{2}, \ldots, w_{m}\right\}$. The generation of $\boldsymbol{s}$ follows the recursive process that input $w_{1: k}$ to the transformer decoder to obtain $w_{k+1}$, then input $w_{1: k+1}$ to compute $w_{k+2}$ and so on. The first input token $w_{1}$ is always the special beginning token [START].

\subsubsection{Encoder Transformer as A Semantic Classifier}

As the generated summary may be off the article theme at the beginning of finetuning, we also optimize TED such that the generated summaries are semantically closed to the input articles. We frame the semantic similarity problem in a discriminative setting. To better adapt to the target-domain data, we add sentence pairs from training articles to facilitate similarity computation.

Concretely, during training, we pick two consecutive sequences of tokens $\boldsymbol{a}_{1}$ and $\boldsymbol{a}_{2}$ from an article to form a positive sequence pair $\left\{\boldsymbol{a}_{1}, \boldsymbol{a}_{2}\right\}$. Second, sequence $\boldsymbol{b}_{1}$ is chosen from another random article in the dataset to form the negative sequence pair $\left\{\boldsymbol{a}_{1}, \boldsymbol{b}_{1}\right\}$. Following Devlin et al. (2018), each sequence pair is packed into one single sequence by inserting a special token [SEP] between them and adding trainable segment embeddings. A special classification token [CLS] is also added to the beginning of the packed sequence. As shown

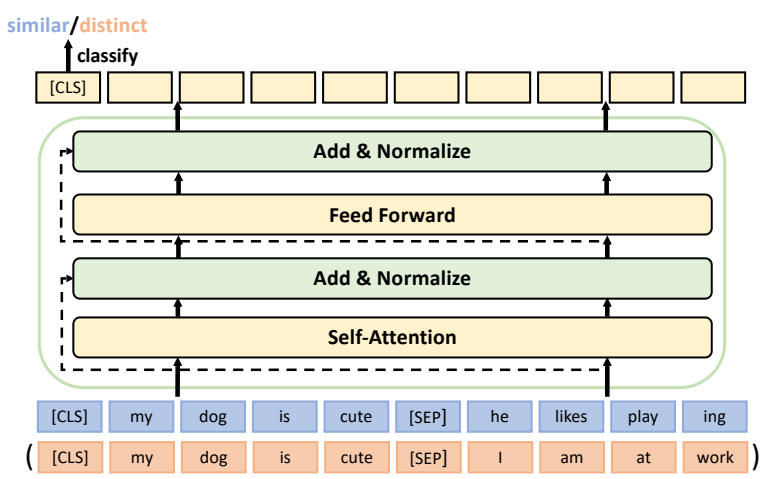

Figure 3: Theme modeling is essentially updating TED with a semantic classifier. The input sentence pair is first processed by adding a "class" token in the beginning and a "separation" token between the two sentences. Then the sentence pair is fed into the transformer encoder, and the first output vector is classified to "similar" or "distinct".

in Fig. 3, the packed sequence is then fed as input into TED's transformer encoder. The output vector associated with the token [CLS], is then classified into similar/distinct categories by a two-layer fully connected network. We use the following crossentropy loss to optimize the encoder such that the $\boldsymbol{a}_{1}$ is semantically similar to $\boldsymbol{a}_{2}$ and $s$ is also closed to $\boldsymbol{d}$, while $\boldsymbol{a}_{1}$ is semantically distinct from $\boldsymbol{b}_{1}$.

$$
\begin{aligned}
& \mathcal{L}_{\text {theme }}=-\log \left(p\left(y=1 \mid \boldsymbol{a}_{1}, \boldsymbol{a}_{2}\right)\right) \\
& \quad-\log (p(y=1 \mid \boldsymbol{s}, \boldsymbol{d}))-\log \left(p\left(y=0 \mid \boldsymbol{a}_{1}, \boldsymbol{b}_{1}\right)\right)
\end{aligned}
$$

\subsection{Denoising Autoencoder}

The idea of denoising autoencoder (Vincent et al., 2008) has been used in unsupervised machine translation (Artetxe et al., 2017; Lample et al., 2017) to prevent the model from learning to merely copy every input word one by one. This denoising process imitates text simplification and helps to refine essential semantic information.

In detail, a sequence of $n$ consecutive tokens $\boldsymbol{x}$ from the input article is injected with two types of noise. First, we insert noisy tokens sampled from other articles in the same dataset into the original sequence at random positions, obtaining a new sequence with length $n^{\prime}$, where $n^{\prime}$ is $40 \%-50 \%$ larger than $n$. Next, similar to Lample et al. (2017), the sequence is slightly shuffled by applying a permutation $\sigma$ such that $\forall i \in\left[1,2, \cdots, n^{\prime}\right],|\sigma(i)-i| \leq k$, where the permutation distance $k$ is set to be $20 \%$ of the length of $\boldsymbol{x}$. The final corrupted sequence is denoted as $\boldsymbol{x}^{\prime}$. TED model is then trained to 
recover the original token sequence given the corrupted sequence:

$$
\mathcal{L}_{\text {denoise }}=C E\left(\boldsymbol{x}, \operatorname{TED}\left(\boldsymbol{x}^{\prime}\right)\right)
$$

where $C E$ denotes the mean of token-level crossentropy loss. $\operatorname{TED}\left(\boldsymbol{x}^{\prime}\right)$ denotes the sequence of probability distribution outputs $\{\boldsymbol{\pi}\}$ from the decoder with inputting $\boldsymbol{x}^{\prime}$ to the encoder. The final objective function is the mean of Eq. (3) and Eq. (4) (we empirically find that equal weights between the two terms work well enough in practice):

$$
\mathcal{L}_{\mathrm{TED}}=\frac{\mathcal{L}_{\text {theme }}+\mathcal{L}_{\text {denoise }}}{2}
$$

It is worth pointing out that we do not conduct "pretraining" on target evaluation datasets. This is because for a target dataset, we do not know beforehand whether the Lead-X sentences will make a quality summary or not. We do have the option to do so on datasets where Lead-X are good summaries, however, it is potentially cherry-picking datasets. Also, we do not conduct supervised finetuning with ground-truths summaries in evaluation datasets because we want to have an entirely unsupervised summarization system with motivations stated in the introduction section.

\section{Experiments}

\subsection{Datasets}

We evaluate our model on three benchmark summarization datasets: NYT, CNN/DM and English Gigaword, containing $110 \mathrm{~K}, 300 \mathrm{~K}$ and $3.8 \mathrm{M}$ news articles, respectively. The detailed statistic information on the datasets can be found in the appendix. In NYT, following Liu and Lapata (2019), we choose 4,000 examples as the validation set and filter out examples with summaries of fewer than 50 words. In CNN/DM, similar to See et al. (2017) and Liu and Lapata (2019), input articles are truncated to 500 tokens. In English Gigaword, we filter out data examples with articles containing only"UNK" tokens.

\subsection{Baseline and Metrics}

We compare TED with the following baselines. (1) Unsupervised abstractive systems: Brief (Wang and Lee, 2018), SEQ ${ }^{3}$ (Baziotis et al., 2019), GPT2 (Radford et al. (2019), without supervised finetuning with ground-truths summaries). (2) Unsupervised extractive systems: TextRank (Mihalcea and Tarau, 2004), Lead-X. (3) Supervised abstractive and abstractive (models trained with groundtruths summaries): PACSUM (Zheng and Lapata, 2019), PGNet (See et al., 2017), REFRESH (Narayan et al., 2018) and SUMO (Liu et al., 2019b). TED is unsupervised abstractive and therefore not directly comparable with supervised baselines. The purpose of supervised systems here is for references. We describe the implementation details of our model in Appendix. We measure the quality of generated summaries by ROUGE F1 score (Lin, 2004), including unigram (ROUGE-1), bigram (ROUGE-2) and longest common subsequence (ROUGE-L).

\subsection{Results}

Results on English Gigaword dataset are shown in Table 2, TED outperforms all unsupervised baselines. Table 2 shows the experimental results on NYT and CNN/DM datasets. In NYT, the unsupervised fine-tuning of TED improves upon the pretrained model by $2.75 \% / 1.06 \% / 2.37 \%$ on ROUGE1/ROUGE-2/ROUGE-L respectively. Note that ROUGE metric prefers extractive systems that preserve original phrasing (See et al., 2017). Considering this factor, TED achieves results that are competitive with unsupervised extractive baselines and surpasses all unsupervised abstractive models. In CNN/DM, TED with a larger model size (10L8H) outperforms all unsupervised abstractive methods and compares favorably with unsupervised extractive baselines. Note that TED outperforms GPT-2, a powerful transformer-based language generation model pretrained on large scale webpage textual data, by significant margins. Again, TED further improves upon pretrained models on both $10 \mathrm{~L} 8 \mathrm{H}$ and $4 \mathrm{~L} 4 \mathrm{H}$ configurations.

Table 1: Results on the English Gigaword dataset. Performances of baseline models are collected from their original papers. The best performance in each metric is in bold.

\begin{tabular}{l|ccc}
\hline Model & $\mathrm{R} 1$ & $\mathrm{R} 2$ & $\mathrm{RL}$ \\
\hline TED 10L8H (ours) & $\mathbf{2 5 . 5 8}$ & $\mathbf{8 . 9 4}$ & $\mathbf{2 2 . 8 3}$ \\
Pretrained 10L8H (ours) & 25.23 & 8.84 & 22.56 \\
TED 4L4H (ours) & 24.59 & 8.10 & 21.91 \\
Pretrained 4L4H (ours) & 22.52 & 7.46 & 20.09 \\
LEAD-8 $^{3}$ & 21.86 & 7.66 & 20.45 \\
BEQ $^{3}$ & 25.39 & 8.21 & 22.68 \\
Brief & 21.26 & 5.60 & 18.89 \\
\hline
\end{tabular}


Table 2: ROUGE $F_{1}$ scores on CNN/DM and NYT datasets. R1/R2/RL stands for ROUGE-1/ROUGE-2/ROUGE$\mathrm{L}$ respectively. Best results in each unsupervised category is in bold. Results of baseline models are obtained from their original papers or running open-sourced codes.

\begin{tabular}{l|ccc|ccc}
\hline & \multicolumn{3}{c}{ CNN/DM } & NYT \\
\hline Model & R1 & R2 & RL & R1 & R2 & RL \\
\hline \multicolumn{5}{c}{ Unsupervised Abstractive } \\
\hline TED 10L8H (ours) & $\mathbf{3 8 . 7 3}$ & $\mathbf{1 6 . 8 4}$ & $\mathbf{3 5 . 4 0}$ & $\mathbf{3 7 . 7 8}$ & $\mathbf{1 7 . 6 3}$ & $\mathbf{3 4 . 3 3}$ \\
Pretrained 10L8H (ours) & 38.38 & 16.49 & 35.08 & 35.03 & 16.57 & 31.96 \\
TED 4L4H (ours) & 34.38 & 9.56 & 30.10 & 24.45 & 7.97 & 21.77 \\
Pretrained 4L4H (ours) & 31.20 & 10.05 & 27.80 & 22.56 & 7.38 & 18.79 \\
SEQ ${ }^{3}$ & 23.24 & 7.10 & 22.15 & 17.85 & 3.94 & 19.53 \\
Brief & 28.11 & 9.97 & 25.41 & - & - & - \\
GPT-2 & 29.34 & 8.27 & 26.58 & - & - & - \\
\hline \multicolumn{7}{c}{ Unsupervised Extractive } \\
\hline LEAD-3 & 40.50 & 17.70 & 36.70 & 35.50 & 17.20 & 32.00 \\
TextRank + tf-idf & 33.20 & 11.80 & 29.60 & 33.20 & 13.10 & 29.00 \\
TextRank + skip-thought & 31.40 & 10.20 & 28.20 & 30.10 & 9.60 & 26.10 \\
TextRank + BERT & 30.80 & 9.60 & 27.40 & 29.70 & 9.00 & 25.30 \\
PACSUM + tf-idf & 39.20 & 16.30 & 35.30 & 40.40 & 20.60 & 36.40 \\
PACSUM + skip-thought & 38.60 & 16.10 & 34.90 & 38.30 & 18.80 & 34.50 \\
PACSUM + BERT & $\mathbf{4 0 . 7 0}$ & $\mathbf{1 7 . 8 0}$ & $\mathbf{3 6 . 9 0}$ & $\mathbf{4 1 . 4 0}$ & $\mathbf{2 1 . 7 0}$ & $\mathbf{3 7 . 5 0}$ \\
\hline \multicolumn{7}{c}{ Supervised Abstractive \& Extractive } \\
\hline SUMO & 41.00 & $\mathbf{1 8 . 4 0}$ & 37.20 & 42.30 & $\mathbf{2 2 . 7 0}$ & $\mathbf{3 8 . 6 0}$ \\
PGNet & 39.50 & 17.30 & 36.40 & $\mathbf{4 2 . 7 0}$ & 22.10 & 38.00 \\
REFRESH & $\mathbf{4 1 . 3 0}$ & $\mathbf{1 8 . 4 0}$ & $\mathbf{3 7 . 5 0}$ & 41.30 & 22.00 & 37.80 \\
\hline
\end{tabular}

\footnotetext{
Article:

after exposing potential security risks with airlines' in-flight entertainment systems, one of the top experts on counter-threat intelligence in the world was pulled off a flight by fbi agents. chris roberts, who featured in a string of fox news reports, was yanked off his plane after it landed in syracuse, new york, on wednesday night by two fbi agents and two uniformed officers. roberts, who works for security intelligence company one world labs, was questioned for the next four hours ...

TED Summary:

chris roberts, who works for security intelligence company one world labs, was pulled off a plane in syracuse, new york, on wednesday night by two fbi agents and two uniformed officers. the incident occurred only a few hours after a report about roberts' research was released by the government accountability office earlier this week.

\section{Reference:}

chris roberts of one world labs grabbed after plane landed in syracuse. two fbi agents spent four hours questioning him about cyberhacking. agents confiscated electronic devices and computer files from roberts. he flew in to give talk at aerospace conference about plane vulnerabilities. roberts featured on fox news' on the record with greta van susteren. regarded as one of the world's top experts on counter-threat intelligence."
}

Figure 4: An example of a generated summary by TED. The reference summary and parts of the input article are also included. 


\section{Discussion}

\subsection{Ablation Study}

The ablation studies shown in Table 3 verify the effectiveness of each component in TED. Training the transformer encoder-decoder from scratch yields reasonable performance. Pretraining on large-scale data results in more than $10 \%$ improvement on all three metrics on training TED from scratch. Pretraining plus either theme modeling or denoising improves upon the pretrained model by more than $2 \%$. The full TED model, pretraining with theme modeling and denoising, produces the best result overall.

Table 3: Ablation study of different components in TED on the NYT dataset. We test with the $10 \mathrm{~L} 8 \mathrm{H}$ model configuration.

\begin{tabular}{l|ccc}
\hline Model & $\mathrm{R} 1$ & $\mathrm{R} 2$ & $\mathrm{RL}$ \\
\hline Train from scratch & 24.49 & 4.41 & 20.14 \\
Pretrained only & 35.03 & 16.57 & 31.96 \\
Pretrained w/ theme modeling & 37.16 & 18.18 & 34.15 \\
Pretrained w/ denoise loss & 37.48 & 17.83 & 34.05 \\
Full model & 37.78 & 17.63 & 34.33 \\
\hline
\end{tabular}

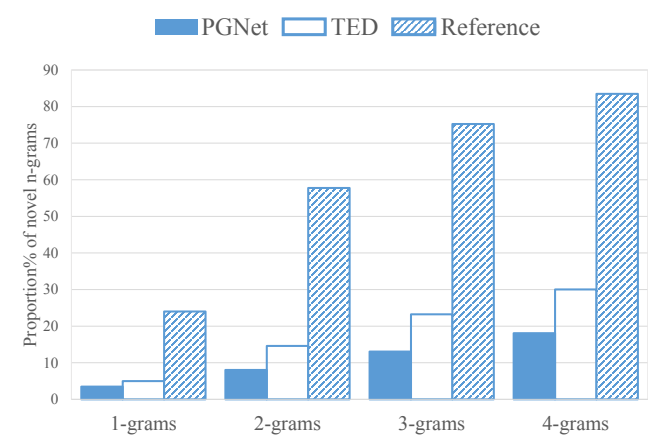

Figure 5: Proportion of novel grams in summaries generated by different models on the CNN/DM test set.

\subsection{Model Analysis}

Example. We showcase a sample summary from CNN/DM dataset along with the input article and the reference summary (Fig. 4). As shown, TED is able to capture and organize the essential information into fluent and highly readable language. We attribute the grammatical correctness to the pretraining process and the denoising autoencoder. However, we also note that although TED manages to recognize the temporal information related to reported event (a few hours after Fox news reports), it makes a mistake by summarizing as "a few hours after a report about roberts' research was released...". It shows that fact cross-checking is a potential future research direction.

Abstractiveness. To examine how abstractive TED is, we compute the proportion of novel Ngrams in the summary output (Fig. 5). The reference summary and the output from PGNet are included for comparison. Although TED is unsupervised, it includes more novel grams than the supervised model PGNet. The reference summaries have the highest proportion of $n$-grams.

\subsection{Comparison with Previous Unsupervised Models}

TED is an innovative unsupervised summarization model with several distinctive features setting it apart from previous approaches such as MeanSum and $\mathrm{SEQ}^{3}$. First, TED leverages the structure of news articles for an effective large-scale pretraining. Second, although both MeanSum and SEQ ${ }^{3}$ have a loss to make the summary similar to the input article, they leverage the classical cosine similarity on text embeddings. In contrast, TED innovatively encodes the similarity by a transformer encoder with much more modeling capability. Third, the denoising module in TED is completely distinct from the idea of reconstruction in $\mathrm{SEQ}^{3}$ and MeanSum. In TED's denoising module, the corrupted texts are input to the transformer and the model is trained to filter the added noises. The original clean document is not used as input and thus unseen by TED in the forward pass. However, the reconstruction process in MeanSum and $\mathrm{SEQ}^{3}$ employs the original document to generate a summary, which is then used to reconstruct the original document.

\section{Conclusion}

In this paper, we propose TED, an unsupervised abstractive summarization model. First, we introduce an effective large-scale pretraining approach leveraging the lead bias in news articles. The pretraining employs automatic filtering mechanism and does require any human-labeled data. We then develop a finetuning scheme to induce the semantic similarity between summaries and input articles, together with a denoising autoencoder to improve the quality of generated summaries. Experiments across three datasets show that TED significantly outperforms unsupervised abstractive baselines. 


\section{References}

Mikel Artetxe, Gorka Labaka, Eneko Agirre, and Kyunghyun Cho. 2017. Unsupervised neural machine translation. arXiv preprint arXiv:1710.11041.

Christos Baziotis, Ion Androutsopoulos, Ioannis Konstas, and Alexandros Potamianos. 2019. Seq 3: Differentiable sequence-to-sequence-to-sequence autoencoder for unsupervised abstractive sentence compression. arXiv preprint arXiv:1904.03651.

Sergey Brin and Lawrence Page. 1998. The anatomy of a large-scale hypertextual web search engine. In COMPUTER NETWORKS AND ISDN SYSTEMS, pages 107-117. Elsevier Science Publishers B. V.

Eric Chu and Peter J Liu. 2018. Meansum: A neural model for unsupervised multi-document abstractive summarization. arXiv preprint arXiv:1810.05739.

Jacob Devlin, Ming-Wei Chang, Kenton Lee, and Kristina Toutanova. 2018. Bert: Pre-training of deep bidirectional transformers for language understanding. arXiv preprint arXiv:1810.04805.

Li Dong, Nan Yang, Wenhui Wang, Furu Wei, Xiaodong Liu, Yu Wang, Jianfeng Gao, Ming Zhou, and Hsiao-Wuen Hon. 2019. Unified language model pre-training for natural language understanding and generation. arXiv preprint arXiv:1905.03197.

Thibault Févry and Jason Phang. 2018. Unsupervised Sentence Compression using Denoising AutoEncoders. arXiv e-prints, page arXiv:1809.02669.

Beliz Gunel, Chenguang Zhu, Michael Zeng, and Xuedong Huang. 2020. Mind the facts: Knowledgeboosted coherent abstractive text summarization. arXiv preprint arXiv:2006.15435.

Karl Moritz Hermann, Tomas Kocisky, Edward Grefenstette, Lasse Espeholt, Will Kay, Mustafa Suleyman, and Phil Blunsom. 2015. Teaching machines to read and comprehend. In Advances in neural information processing systems, pages 1693-1701.

Eric Jang, Shixiang Gu, and Ben Poole. 2016. Categorical reparameterization with gumbel-softmax. arXiv preprint arXiv:1611.01144.

Taku Kudo and John Richardson. 2018. Sentencepiece: A simple and language independent subword tokenizer and detokenizer for neural text processing. arXiv preprint arXiv:1808.06226.

Guillaume Lample, Alexis Conneau, Ludovic Denoyer, and Marc' Aurelio Ranzato. 2017. Unsupervised machine translation using monolingual corpora only. arXiv preprint arXiv:1711.00043.

Chin-Yew Lin. 2004. Rouge: A package for automatic evaluation of summaries. In Text summarization branches out, pages 74-81.
Liyuan Liu, Haoming Jiang, Pengcheng He, Weizhu Chen, Xiaodong Liu, Jianfeng Gao, and Jiawei Han. 2019a. On the variance of the adaptive learning rate and beyond. arXiv preprint arXiv:1908.03265.

Yang Liu and Mirella Lapata. 2019. Text Summarization with Pretrained Encoders. arXiv e-prints, page arXiv:1908.08345.

Yang Liu, Ivan Titov, and Mirella Lapata. 2019b. Single document summarization as tree induction. In Proceedings of the 2019 Conference of the North American Chapter of the Association for Computational Linguistics: Human Language Technologies, Volume 1 (Long and Short Papers), pages 17451755.

Minh-Thang Luong, Ilya Sutskever, Quoc V Le, Oriol Vinyals, and Wojciech Zaremba. 2014. Addressing the rare word problem in neural machine translation. arXiv preprint arXiv: 1410.8206 .

Bryan McCann, James Bradbury, Caiming Xiong, and Richard Socher. 2017. Learned in translation: Contextualized word vectors. In Advances in Neural Information Processing Systems, pages 6294-6305.

Rada Mihalcea and Paul Tarau. 2004. Textrank: Bringing order into text. In Proceedings of the 2004 conference on empirical methods in natural language processing, pages 404-411.

Shashi Narayan, Shay B Cohen, and Mirella Lapata. 2018. Ranking sentences for extractive summarization with reinforcement learning. arXiv preprint arXiv:1802.08636.

Rodrigo Nogueira and Kyunghyun Cho. 2019. Passage re-ranking with bert. arXiv preprint arXiv:1901.04085.

Matthew E Peters, Mark Neumann, Mohit Iyyer, Matt Gardner, Christopher Clark, Kenton Lee, and Luke Zettlemoyer. 2018. Deep contextualized word representations. arXiv preprint arXiv:1802.05365.

Alec Radford, Karthik Narasimhan, Tim Salimans, and Ilya Sutskever. 2018. Improving language understanding by generative pre-training.

Alec Radford, Jeffrey Wu, Rewon Child, David Luan, Dario Amodei, and Ilya Sutskever. 2019. Language models are unsupervised multitask learners. OpenAI Blog, 1(8).

Abigail See, Peter J Liu, and Christopher D Manning. 2017. Get to the point: Summarization with pointergenerator networks. In Proceedings of the 55th Annual Meeting of the Association for Computational Linguistics (Volume 1: Long Papers), pages 10731083.

Rico Sennrich, Barry Haddow, and Alexandra Birch. 2015. Neural machine translation of rare words with subword units. arXiv preprint arXiv:1508.07909. 
Ilya Sutskever, Oriol Vinyals, and Quoc V Le. 2014. Sequence to sequence learning with neural networks. In Advances in neural information processing systems, pages 3104-3112.

Ashish Vaswani, Noam Shazeer, Niki Parmar, Jakob Uszkoreit, Llion Jones, Aidan N Gomez, Ł ukasz Kaiser, and Illia Polosukhin. 2017. Attention is all you need. In I. Guyon, U. V. Luxburg, S. Bengio, H. Wallach, R. Fergus, S. Vishwanathan, and R. Garnett, editors, Advances in Neural Information Processing Systems 30, pages 5998-6008. Curran Associates, Inc.

Pascal Vincent, Hugo Larochelle, Yoshua Bengio, and Pierre-Antoine Manzagol. 2008. Extracting and composing robust features with denoising autoencoders. In Proceedings of the 25th international conference on Machine learning, pages 1096-1103. ACM.

Yau-Shian Wang and Hung-Yi Lee. 2018. Learning to encode text as human-readable summaries using generative adversarial networks. arXiv preprint arXiv:1810.02851.

Zichao Yang, Zhiting Hu, Chris Dyer, Eric P Xing, and Taylor Berg-Kirkpatrick. 2018. Unsupervised text style transfer using language models as discriminators. In Advances in Neural Information Processing Systems, pages 7287-7298.

Ziyi Yang, Chenguang Zhu, Vin Sachidananda, and Eric Darve. 2019. Embedding imputation with grounded language information. arXiv preprint arXiv:1906.03753.

Hao Zheng and Mirella Lapata. 2019. Sentence centrality revisited for unsupervised summarization. arXiv preprint arXiv:1906.03508.

Chenguang Zhu, Michael Zeng, and Xuedong Huang. 2018. Sdnet: Contextualized attention-based deep network for conversational question answering. arXiv preprint arXiv:1812.03593. 